\title{
Trends in prevalence of allergic diseases in Korean children: how and why?
}

Kyung Suk Lee, MD, PhD

Department of Pediatrics, Hanyang University Guri Hospital, Hanyang University College of Medicine, Guri, Korea

The prevalence of allergic diseases is an important issue in the medical field. ${ }^{1)}$ Asthma, allergic rhinitis, and atopic dermatitis influence children's health and quality of life and increase their socioeconomic burden. ${ }^{1)}$ Previous studies have analyzed the prevalence of these allergic diseases to institute prevention and treatment protocols. The International Study of Asthma and Allergies in Childhood (ISAAC), the representative global study of the prevalence of allergic diseases, conducted phases I-IV in 1991-2012. ${ }^{2)}$ Prototypal questionnaires in this study are utilized to define allergic diseases in prevalence studies. ${ }^{2)}$ In Korea, the ISAAC has been ongoing since the 1990s, and the data were published in a report by the ISAAC Steering Committee in 1998. ${ }^{3)}$ National health surveys such as the Korea National Health and Nutrition Examination Survey and Korea Youth Risk Behavior Web-based Survey have also investigated the prevalence of allergic diseases. ${ }^{4)}$

The incidence of allergic diseases has been gradually increasing over the past few decades, especially in developing countries. ${ }^{5)}$ However, according to the study by $\mathrm{Ha}$ et al., ${ }^{6}$ the trend in recent prevalence is changing. Asthma, a relatively severe respiratory allergic disease, has been decreasing in children and the elderly, whereas allergic rhinitis has been increasing in all age groups above school-age children. ${ }^{6}$ The trend in prevalence of atopic dermatitis varies widely among age groups. ${ }^{6}$ This shift in the trend suggests that, although the treatment of severe allergic diseases such as asthma is important, we should also pay more attention to managing mild allergic diseases such as allergic rhinitis that impact quality of life.

The Korean central and local governments' active policy against allergic diseases is one reason for the decrease in asthma. ${ }^{\text {) }}$ For example, the Atopic Asthma Education Information Center and Atopy-Asthma-Friendly School Project aims to actively inform Koreans about disease prevention and management. ${ }^{7)}$ The most distinctive argument in the study by $\mathrm{Ha}$ et al. ${ }^{6}$ is that the withdrawal of humidifier disinfectants had a significant effect on reducing asthma rates. In Korea, from 1994 to 2001, the extensive use of humidifier disinfectants was a serious risk factor that increased the prevalence of respiratory diseases including asthma and lung injury.8)

On the contrary, allergic rhinitis has shown interesting increase in prevalence. $\left.{ }^{6}\right)$ Humidifier disinfectant use is also a risk factor for allergic rhinitis; demonstrations in rat experiments showed degeneration of the normal nasal epithelium. $\left.{ }^{9}\right)$ However, with the decreasing use of humidifier disinfectants, the prevalence of allergic rhinitis has increased while that of asthma has decreased, which defies the concept of a one-airway disease. ${ }^{6}$ Further research is needed to ascertain this opposing change in the prevalence of asthma and allergic rhinitis. In addition, the $\mathrm{Ha}$ et al. ${ }^{6}$ study indicated that environmental pollution, climate change, and especially the exposure to chemicals such as phthalate are crucial factors in the development of allergic rhinitis. Accordingly, management at the government level is important to control such risk factors of allergic diseases.

The prevalence of atopic dermatitis has decreased in infants and preschool children but increased in the elderly age group. ${ }^{6}$ In Korea, the increase in breast milk feeding, vaginal delivery, and probiotics consumption might have contributed to the decrease in the prevalence of atopic dermatitis in the younger age group. ${ }^{10)}$ However, further studies are required to determine the causes of the increasing incidence of atopic dermatitis in elderly individuals.

In conclusion, the trends in the prevalence of allergic diseases vary among diseases and age groups. ${ }^{6}$ An analysis of the underlying causes of allergic diseases is important to formulating countermeasures for such diseases and will provide insight into these trends over the next decade.

\section{Conflicts of interest}

No potential conflict of interest relevant to this article was reported.

See the article "Ten-year trends and prevalence of asthma, allergic rhinitis, and atopic dermatitis among the Korean population, 2008-2017" via https://doi.org/10.3345/cep.2019.01291.

\footnotetext{
Corresponding author: Kyung Suk Lee, MD, PhD. Department of Pediatrics, Hanyang University Guri Hospital, Hanyang University College of Medicine, 153 Gyeongchun-ro, Guri 11923, Korea 


\section{References}

1. Baiardini I, Braido F, Brandi S, Canonica GW. Allergic diseases and their impact on quality of life. Ann Allergy Asthma Immunol 2006;97:419-28

2. The International Study of Asthma and Allergies in Childhood [Internet]. International Study of Asthma and Allergies in Childhood; c1991 [cited 2020 Feb 12]. Available from: http://isaac.auckland.ac.nz/index.html.

3. Worldwide variation in prevalence of symptoms of asthma, allergic rhinoconjunctivitis, and atopic eczema: ISAAC. The International Study of Asthma and Allergies in Childhood (ISAAC) Steering Committee. Lancet 1998;351:1225-32.

4. Korea Centers for Disease Control \& Prevention. Coronavirus disease 19 [Internet]. Cheongju (Korea): Korea Centers for Disease Control \& Prevention; c2019 [cited 2020 Mar 30]. Available from: http://www.cdc. go.kr/index.es? sid $=\mathrm{a} 2$.

5. Pawankar R. Allergic diseases and asthma: a global public health concern and a call to action. World Allergy Organ J 2014;7:12.
6. Ha J, Lee SW, Yon DK. Ten-year trends and prevalence of asthma, allergic rhinitis, and atopic dermatitis among the Korean population, 2008-2017. Clin Exp Pediatr 2020;63:278.

7. Cho YM, Kim CB, Yeon KN, Lee ES, Kim K. Trends in the prevalence of childhood asthma in Seoul Metropolitan City, Korea: the seoul atopy asthma-friendly school project. J Prev Med Public Health 2018;51:27580.

8. Yoon J, Lee SY, Lee SH, Kim EM, Jung S, Cho HJ, et al. Exposure to humidifier disinfectants increases the risk of asthma in children. Am J Respir Crit Care Med 2018;198:1583-6.

9. Koh TH, Park JT, Kwon D, Kwak K. Association between use of humidifier disinfectant and allergic rhinitis in Korean children: a crosssectional study based on the eighth Panel Study on Korean Children (PSKC). Ann Occup Environ Med 2020;32:e9.

10. Lee JY, Yang HK, Kim M, Kim J, Ahn K. Is the prevalence of atopic dermatitis in Korean children decreasing? National Database 2009-2014. Asian Pac J Allergy Immunol 2017;35:144-9. 